\title{
Horizontal and vertical distance perception: The discorded-orientation theory
}

\author{
ATSUKI HIGASHIYAMA \\ University of Osaka Prefecture, Sakai, Japan
}

\begin{abstract}
We sought the conditions where the horizontal-vertical illusion (HVI) takes place outdoors in an open field. Longitudinal distance from a subject to a building wall was adjusted to appear equal to the vertical or horizontal distance on the wall. To examine validity of previous theories (physiology, frame, depth, and gravity theories), boundary of visual field (ellipse and circle), bodily orientation (upright and lying), and orientation of visual objects (normal, $90^{\circ}$-tilted, and inverse) were manipulated in eight experiments. These three independent variables affected the HVI effects, but their effects were not explained by the previous theories. We therefore proposed a model on the basis of discord among the retinal, visual, and gravitational orientations. We also found that longitudinal distance was adjusted as being consistently larger than the standard distance. This result was explained by the reduction of cues to distance and the HVI effect.
\end{abstract}

When seeing an object such as a building, we should distinguish among longitudinal, vertical, and horizontal distances. By longitudinal distance, we mean distance from an observer to the target. By vertical distance, we mean height of the target from the ground. By horizontal distance, we mean lateral width of the target. The term frontal distance is used to represent both vertical and horizontal distances. The term depth is also used to refer to a spatial separation between two points, positioned on the same visual direction.

The main concern of this study was to clarify how the relation between apparent vertical and horizontal distances is determined, by adjusting the longitudinal distance so that it appears equal to the frontal distance. This issue has been studied as the horizontal-vertical illusion (HVI). We first reviewed four previous theories of the HVI. We next demonstrated that the previous theories fail to explain the results of the eight experiments in this study and proposed an alternative theory that is based on discord among retinal, visual, and gravitational orientations.

We also found that longitudinal distance was adjusted to be consistently larger than the standard frontal distance. This finding is discussed in relation to the size-distance invariance hypothesis, discord among the spatial orientations, and reduction of cues to distance.

\section{Previous Theories}

The HVI is a tendency to perceive a vertical line as being longer than a horizontal line of the same objective

This study was supported in part by Grants-in-Aid for Scientific Research (No.06610080) provided by the Japanese Ministry of Education, Science, and Culture. The author is grateful to H. A. Sedgwick and $\mathbf{K}$. Shimono for their critical reading of an earlier manuscript and to M. L. Braunstein for his editorial assistance. Correspondence should be addressed to A. Higashiyama, Institute of Human Sciences, College of Integrated Arts and Sciences, The University of Osaka Prefecture, Sakai 593, Japan (e-mail: achan@center.osakafu-u.ac.jp). size. There are several theories concerning the HVI. The first is a physiological explanation in which the neuroanatomy of the retina, the detectors in the brain, the dioptrics of the cornea, and/or eye movement are thought to be important components in producing the HVI. Assuming greater density of retinal receptors in the horizontal rather than vertical meridian, Ritter (1917) suggested that this asymmetric organization of the retina accounts for the overestimation of the vertical line in the HVI figure. Caelli (1977) predicted the HVI from the interaction between detectors making selective responses to orientation. Valentine (1912a) and Pearce and Matin (1969) ascribed the HVI to the effects of corneal astigmatism. At the region of the optical axis, the corneal surface is spherical and is not distortive, but as the corneal surface approaches the sclera, the vertical meridian is strongly curved relative to its horizontal meridian. It follows that the average curvature for the vertical meridian is greater than that for the horizontal meridian. Wundt (1858, cited in Boring, 1942, and Luckiesh, 1965) maintained that because of the arrangement of eye muscles, more effort is required to raise the eyes through a vertical distance than through an equal horizontal distance. If we appraise the effort of the muscles in terms of distance, the vertical distance would be perceived as larger than the horizontal distance.

The common feature of these physiological explanations is an increase in the magnification of the vertical meridian relative to the horizontal meridian. Although the effect of each physiological component may be small, these components would accumulate to explain the HVI (Thompson \& Schiffman, 1974a).

On the basis that the more a target approaches its surrounding frame, the larger the target appears (Künnapas, 1955; Restle, 1970; Restle \& Merryman, 1969), Künnapas (1957a, 1957b, 1957c) ascribed the HVI effect to the horizontally elliptical frame of the visual field. For the 
monocular visual field, the vertical extent is about $135^{\circ}$ and the horizontal extent is about $160^{\circ}$ (Henson, 1993). For the binocular visual field, the horizontal extent is about $200^{\circ}$ with a $60^{\circ}$ overlapping portion of the monocular fields, and the vertical extent remains the same as the monocular vertical extent. Thus, regardless of monocular or binocular observation, the vertical line looks larger than the horizontal line because the vertical line is closer to the visual field boundary than is the horizontal line.

The frame theory has several predictions about the HVI, but the evidence in support of these predictions seems contradictory. First, binocular vision should produce greater illusion than monocular vision, because the ratio of the vertical to horizontal extents is smaller in the binocular than in the monocular visual field. Prinzmetal and Gettleman (1993) confirmed this prediction, but Valentine (1912b) and Künnapas (1957a) did not. Second, in well-lit situations where the visual field boundary is perceived clearly, the illusion should augment as compared with dark situations where the visual frame disappears (Künnapas, 1957c; Prinzmetal \& Gettleman, 1993; Verrillo \& Irvin, 1979). Yet, Avery and Day (1969) found no difference in effect of the illusion among dark, semidark, and light situations. Third, the illusion would be reversed if subjects tilted their heads $90^{\circ}$ from the vertical: The horizontal line appears larger than the vertical line of the same objective size. Avery and Day (1969) and Künnapas (1958) discovered such a reversed effect. However, Prinzmetal and Gettleman (1993) did not find it, although the illusion effect decreased when it was observed with the head tilted. Last, the direction and magnitude of the illusion are supposed to depend on the artificial field of various shapes surrounding a test target. A horizontally oriented frame facilitated the illusion, whereas a vertically oriented frame reduced the illusion (Künnapas, 1957b, 1959; Prinzmetal \& Gettleman, 1993). However, Houck, Mefferd, and Greenstein (1972) provided evidence against the frame effect: A vertically oriented frame facilitated the illusion and a horizontally oriented frame reduced the illusion. Thompson and Schiffman (1974b) showed that visual frame is not a primary determinant of the HVI.

The third explanation is based on depth cues represented in a two-dimensional picture plane (von Collani, 1985; Coren \& Girgus, 1977, 1978; Cormack \& Cormack, 1974; Girgus \& Coren, 1975; Gregory, 1963, 1972; Schiffman \& Thompson, 1975). The proponents of this theory suggested that a horizontal line in the picture plane does not indicate depth, but a vertical line frequently depicts a line receding into depth, making the top of the vertical line seem more distant from the observer than its bottom. If the retinal image sizes of the lines are converted into apparent sizes by taking the implied depth into account, then the vertical line appears larger than the horizontal line of the same objective size. In short, the vertical line is likely to be seen as representing a foreshortened line extending into depth, whereas the horizontal line is seen as representing a line normal to the line of sight. The depth theory seems to assume that the mechanism scaling depth implied for the vertical line also works for trees and buildings in natural environments, because the HVI was observed for such objects (Chapanis \& Mankin, 1967).

In most studies supporting the depth theory, the retinal vertical agreed with the visual vertical. Accordingly, the definition of vertical was not always clear. If the retinal vertical were important for the HVI, the depth theory would have the same predictions as the physiology theory. Therefore, the visual vertical should be assumed to be crucial in the depth theory. For example, if we lie on our sides, then a tree (i.e., an object suggesting the vertical) is projected on the horizontal retinal meridian and a $\log$ on the ground (i.e., an object suggesting the horizontal) is projected on the vertical retinal meridian. In this case, it is predicted that the tree is perceived to be larger than the log of the same objective size.

Another prediction from the depth theory is that the illusion augments as the depth cues are reduced. This prediction is in contrast with that of the frame theory. In full depth-cue situations, we can easily recognize a line drawn on a plane surface, so that the implied depth is in conflict with the cues suggesting flatness in the picture. However, in reduced depth-cue situations, the implied depth is likely to operate without such a conflict, so that the receding of the vertical line into depth is emphasized. Nevertheless, evidence (Verrillo \& Irvin, 1979) did not support this prediction.

The last possible explanation is the gravity theory, in which a line in parallel with gravity is perceived as larger than a line of the same size that lies perpendicular to gravity. This explanation is based on evidence of the muscular effort needed for locomotion. More effort is made in climbing slopes and staircases than in walking horizontally on the ground. If muscular effort is transformed into apparent distance, then vertical locomotion distance is perceived to be larger than the horizontal locomotion distance. There are no leading advocates of the gravity theory, but this idea seems to prevail as a commonsense explanation. The role of gravity in the HVI is discussed briefly in textbooks (e.g., Howard \& Templeton, 1966).

\section{GENERAL METHOD}

We report the findings from eight experiments in which a building in an open field was used as the standard target. We used a building because the HVI effect has been shown to be more prominent with large objects outdoors (e.g., Chapanis \& Mankin, 1967) than with geometric drawings in laboratories (see, e.g., Begelman \& Steinfeld, 1967). Fourteen undergraduates were assigned as subjects to each experiment; any subject participated in only a single experiment.

The subject was positioned in front of a three-story white building. Figure 1 shows a photograph of the frontal view of the building; its sketch was also provided by Higashiyama (1992). The wall of the building $(12.7 \mathrm{~m}$ tall $\times 30.0 \mathrm{~m}$ wide) was flat, with a row of 15 windows $(176 \mathrm{~cm}$ tall $\times 192 \mathrm{~cm}$ wide $)$ on each floor.

Four vertical and four horizontal distances along the building wall were used as standards. The vertical standards were defined as the distances from the building's base to the tops of the rows of windows (the first floor, $2.7 \mathrm{~m}$; the second floor, $6.7 \mathrm{~m}$; the third floor, $10.5 \mathrm{~m}$ ) 


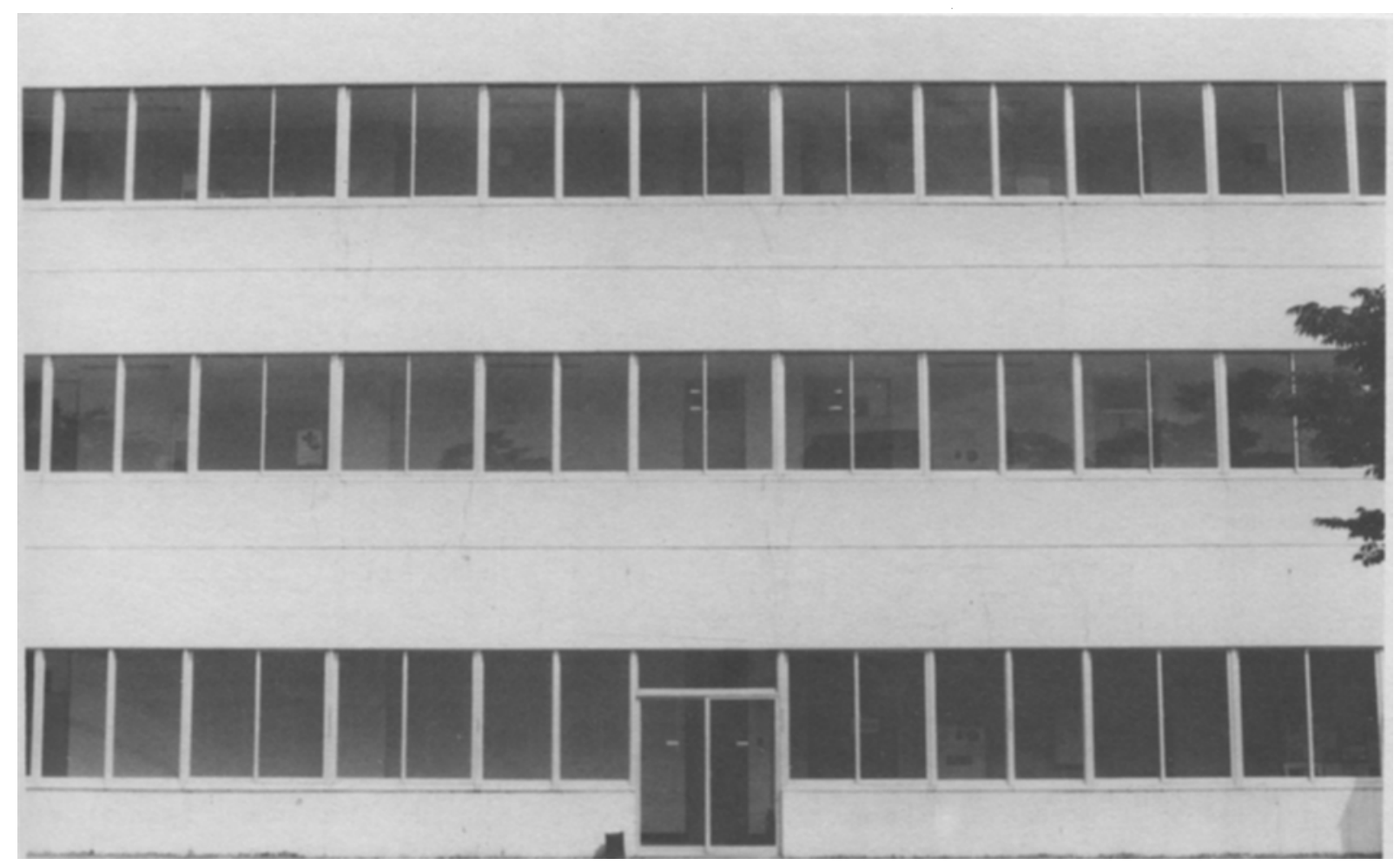

Figure 1. Photograph of the building wall used as the frontal standard distance.

and to the roof of the building $(12.7 \mathrm{~m})$. The horizontal standards were distances of $2,6,9$, and $14 \mathrm{~m}$ from the median plane toward the right. A piece of red tape was pasted at each end of the horizontal standards. The height of the tape was $155 \mathrm{~cm}$ that approximated to the mean eye level of the upright subjects. A measuring tape $(1.4 \mathrm{~cm}$ wide $\times 50 \mathrm{~m}$ long) was placed, from the lower end of the vertical standard to the subject, on the ground. The tape was turned over to prevent the subject from reading the scale of the tape.

In all experiments, the subjects followed the same general procedure. For each standard, the subject was asked to change his/her position along the measuring tape that was perpendicular to the building wall, so that the longitudinal distance from the subject to the wall appeared equal to the standard. The experimenter moved with the subject to measure the longitudinal distance, to an accuracy of $10 \mathrm{~cm}$. A variation of the method of average error was used. The subject made two adjustments, by approaching the building and receding from the building, for each standard. In the approaching trial, the subject started from the position in which the longitudinal distance was definitely perceived to be larger than the standard, and in the receding trial, the subject started from the position in which the longitudinal distance was definitely perceived to be smaller than the standard. The starting position was varied across trials. The subject had unlimited time to make the adjustments and was allowed to make as many adjustments as he/she felt were necessary.

In each experiment, half the subjects made adjustments first for the vertical standards and then for the horizontal standards; the remaining subjects followed the reverse order. For the vertical or horizontal standards, the combinations of four standards and two adjustments were randomly imposed on the subject.

\section{EXPERIMENTS 1-3 Natural Viewing}

Longitudinal distance was adjusted by the upright subjects (Experiment 1 ) and by the subjects lying on their sides (Experiment 2). All of the theories about the HVI predict that when a vertical standard is objectively equal to the horizontal standard, the longitudinal distance adjusted by the upright subjects is larger for the vertical standard than for the horizontal standard. However, for the lying-on-side subjects, the physiology and frame theories predict a reversed HVI effect that the adjustments for the horizontal standard are larger than those for the vertical standard, whereas the depth and gravity theories predict a usual HVI effect that the adjustments for the vertical standard are larger than those for the horizontal standard.

If the HVI effects differ between the upright and lyingon-side conditions, it would be possible to maintain that this difference is a function of head tilt or height of observation. To examine this problem, we had the subjects in Experiment 3 lay on their bellies with their heads erect.

\section{Method}

In Experiment 1, in which the subject stood upright, he/she walked back and forth until the longitudinal distance appeared equal to each standard. In Experiments 2 and 3, the subject got on a cart and adjusted longitudinal distance by changing the position of the cart. The cart consisted of a flat plate $(92 \mathrm{~cm}$ wide $\times 182 \mathrm{~cm}$ long $\times 32 \mathrm{~cm}$ high from the ground) with four wheels $(20 \mathrm{~cm}$ diam.) and was pushed or stopped slowly by the experimenter under the subject's direction.

In Experiment 2, the subject lay on his/her side on the cart, with the head on his/her arm. The cart was moved to keep the subject's body parallel with building wall. Half the subjects directed their bodies to the right and the remaining subjects to the left. The subject was allowed to move his/her head but was asked not to separate the head from the arm used as a head supporter. 
In Experiment 3, the subject lay on his/her belly on the cart, resting the chin in the hands and directing the face to the building. The subject was allowed to move the head but was not allowed to separate the chin from the hands. In Experiments 2 and 3, free eye movement was encouraged.

\section{Results and Discussion}

Figure 2 shows the results: The left panel of Figure 2 is for the upright subjects; the center is for the lying-onside subjects; the right is for the lying-on-belly subjects. In each panel, the abscissa represents the standard distance and the ordinate represents the mean matched distance. The parameter is the standard direction. Each data point represents a mean of 28 adjustments ( 14 subjects $\times 2$ series) and the bar passing through it represents the standard deviation.

For each direction, a least-squares line was individually fitted to the mean longitudinal distance as a function of standard distance. In making this calculation, we assumed that the line should pass through the origin. For the upright subjects, the mean slope for the vertical standard $(1.57, S D=.31)$ was significantly different $[t(13)=$ $9.02, p<.001]$ from the mean slope for the horizontal standard $(1.14, S D=.26)$. For the lying-on-side subjects, the mean slope for the vertical standard $(1.35, S D=$ .21 ) was not significantly different from the mean slope for the horizontal standard $(1.31, S D=.21)$. For the lying-on-belly subjects, the mean slope for the vertical standard $(1.30, S D=.37)$ was significantly different $[t(13)=5.80, p<.001]$ from the mean slope for the horizontal standard $(1.04, S D=.23)$.

None of the extant theories-physiology, frame, depth, or gravity-accounts for all aspects of the results. In particular, no theory explains the absence of difference in slope between the two directions in the lying-on-side condition. In the lying-on-belly condition, the slope for the vertical was steeper than that for the horizontal, whereas in the lying-on-side condition, the two slopes were the same. This finding suggests that a determinant of the HVI is the tilting of the retinal meridians by tilting the head laterally, not the lowering of the height of observation by bending the trunk.

\section{EXPERIMENTS 4-5 Cylindric Viewing}

In Experiments 4-5, the subjects saw the scene monocularly through a cylinder. The boundary of the visual field seen through the cylinder was round, in contrast with the oval boundary under natural binocular viewing. Since the frame theory does not predict the HVI effect for the round boundary, if longitudinal distance is represented as a function of standard distance, then the slope for the vertical standard would be equal to that for the horizontal standard.

Another effect of monocular cylindric viewing is that cues to distance are reduced (Hagen, Jones, \& Reed, 1978;
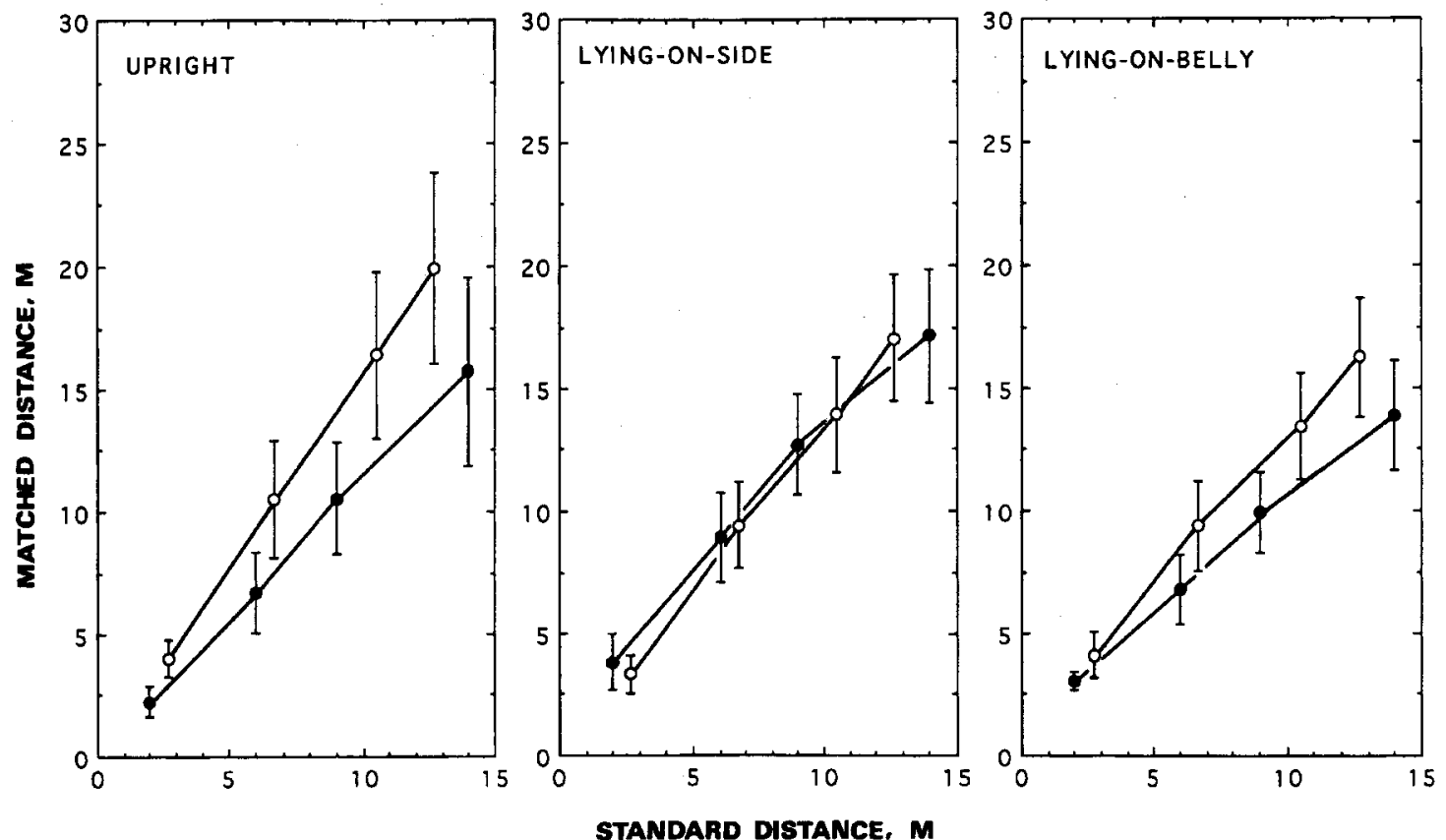

Figure 2. Mean longitudinal distance (in meters) as a function of standard distance under natural binocular viewing. The open circles represent the vertical standard and the filled circles represent the horizontal standard. The left panel is for the upright subjects; the center panel for the lying-on-side subjects; and the right panel for the lying-on-belly subjects. The bars passing through the data points represent the standard deviations. 
Holway \& Boring, 1941). A prediction from the depth theory is that the less the cues to distance, the more the HVI effect.

It seems that in the physiology and gravity theories, viewing the scene through a cylinder would not have any effects on the HVI. Accordingly, in the same way as in the natural binocular condition, the physiology theory predicts the usual HVI for the upright subjects and the reversed HVI for the lying-on-side subjects. Similarly, the gravity theory predicts the usual HVI independently of bodily orientation.

\section{Method}

Experiments 4 and 5 repeated Experiments 1 and 2, respectively, except that a subject viewed monocularly through a cylinder. In both experiments, one of the subject's eyes was covered with a cylinder ( $4 \mathrm{~cm}$ diam. $\times 15 \mathrm{~cm}$ long) and another was occluded by a piece of surgical tape. The cylinder was made by rolling up a piece of black paper $(12.5 \mathrm{~cm}$ wide $\times 15 \mathrm{~cm}$ long) and was held steady on the eye with surgical tape. When seen through the cylinder, the boundary of the visual field subtended a visual angle of $15.2^{\circ}$. Since the monocular visual boundary was considerably smaller than the angular size of the standards, the subjects moved the head up and down (or left and right) to see the ground and the standards. In each experiment, half the subjects wore the cylinder on the left eye and the remaining subjects on the right eye. In Experiment 5 , the subjects who saw only with the left eye lay on their right sides, whereas the subjects who saw only with the right eye lay on their left sides.

\section{Results and Discussion}

Figure 3 shows the mean matched longitudinal distance against standard distance: the left panel for the upright subjects and the right for the lying-on-side subjects. For each direction, a least-squares line passing through the origin was individually fitted to the matched distance as a function of standard distance. For the upright subjects, the mean slope for the vertical standard $(1.71, S D=.37)$ was significantly different $[t(13)=4.46$, $p<.001]$ from the mean slope for the horizontal standard $(1.25, S D=.23)$. For the lying-on-side subjects, the mean slope for the vertical standard $(1.94, S D=.22)$ was not significantly different from the mean slope for the horizontal standard $(1.84, S D=.24)$.

The results of Experiments 4-5 were not consistent with the physiology theory, which predicts an inverted HVI effect for the lying-on-side subjects. Also, these results did not agree with the gravity theory, in which the HVI is assumed to take place independently of bodily orientation. Moreover, these results were not compatible with the frame theory, which assumes that the HVI would not occur within the round visual field.

The HVI effect was not enhanced or reduced with cylindric viewing. In Experiment 4, the slope for the vertical standard was 1.37 times as large as that for the horizontal standard. This ratio was much the same as that in Experiment $1(1.38)$. Likewise, the ratio of the slope for

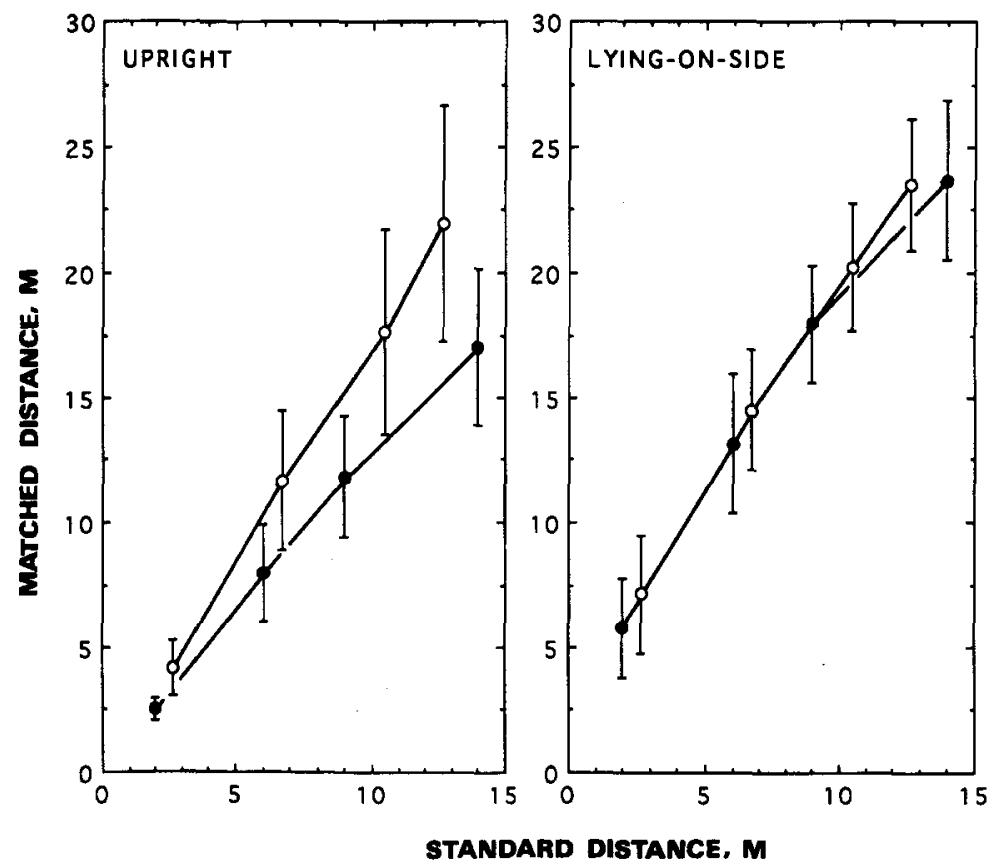

Figure 3. Mean longitudinal distance (in meters) as a function of standard distance under monocular cylindric viewing. The open circles represent the vertical standard and the filled circles represent the horizontal standard. The left panel is for the upright subjects and the right panel for the lying-on-side subjects. The bars passing through the data points represent the standard deviations. 
the vertical to the slope for the horizontal in Experiment 5 was 1.05 , whereas the corresponding slope ratio in Experiment 2 was 1.03. These findings were not compatible with either the depth or the frame theory.

Indeed, the effects of the visual boundary on the HVI seemed weak. Prinzmetal and Gettleman (1993) compared the magnitude of the HVI between binocular and monocular indoor viewings. In an experiment showing the greatest HVI effect, the amounts of illusion (i.e., the percentage difference between matched horizontal and vertical lines) were $7.07 \%$ and $4.87 \%$ for the binocular and monocular viewings, respectively. The difference between the two was only $2.2 \%$, very far from a complete explanation of the HVI.

On the basis that no previous theory explains the results of Experiments 4-5, it may be hypothesized that the complex visual pattern on building wall would produce unexpected extraneous effects on frontal distance perception. However, Higashiyama and Ueyama (1988) demonstrated that the ratio of the longitudinal to vertical distances was about 1.60 consistently for various outdoor objects. Also, there were not great differences between indoor and outdoor situations in constructing the visual alleys (Higashiyama, Ishikawa, \& Tanaka, 1990) and in estimating longitudinal distance (Da Silva, 1985; Higashiyama \& Shimono, 1994).

The mean matches of longitudinal distance under the cylindric viewing were larger than those under the natural binocular viewing. In the upright condition, the slopes for the vertical and horizontal standards in Experiment 4 were 1.09 and 1.10 times as large as those in Experiment 1, respectively. In the lying-on-side condition, the slopes for the vertical and horizontal standards in Experiment 5 were 1.44 and 1.40 times as large as those in Experiment 2, respectively. It is thus suggested that the cylindric viewing affected the matches of longitudinal distance, but not the HVI.

\section{EXPERIMENTS 6-8 Prismatic Viewing}

In Experiments 6-8, the subjects saw the scene through prism goggles that could rotate the retinal image. The purpose of these experiments was to clarify how the visual orientation of the scene affects the HVI. In Experiment 6 , the upright subjects wore goggles that caused the retinal image to be tilted by $90^{\circ}$. In Experiment 7, the subjects, lying on their sides, wore the same goggles. In Experiment 8, the upright subjects wore the goggles by which the retinal image was rotated $180^{\circ}$ around the horizontal axis, thereby producing an inverted retinal image.

Familiar objects such as buildings and trees suggest the visual vertical, regardless of their retinal orientation. Since the depth theory assumes that the orientation suggesting the visual vertical is perceived to be larger, the slope for the vertical standard would be steeper than the slope for the horizontal standard. Thus, the depth theory predicts the usual HVI for Experiments 6-7.
According to the physiology and frame theories, when the retinal image is tilted by $90^{\circ}$, the reversed HVI would be obtained for the upright subjects and the usual HVI would be obtained for lying-on-side subjects. However, the gravity theory predicts the reversed HVI for the upright and lying-on-side subjects, because the vertical standard is orthogonal to the gravity in both bodily orientations.

All previous theories predict the usual HVI for the upright subjects with the retinal images inverted (Experiment 8). Just as the inversion of retinal image yields a drastic change in figure, face, and word perception (Howard, 1982; Köhler, 1940; Rock, 1973), so the inverted scene may result in a change in frontal distance perception. Indeed, Miyagawa (1943) found that size constancy was diminished for the targets that were viewed between the legs with the head upside down, but it was not diminished for the optically inverted targets that were viewed with the upright heads. Rock and Kaufman (1962) showed that the moon illusion was reduced by inverting the scene, and they assumed that apparent distance to the horizontal moon was less with inversion than without inversion. Even if their assumption were applicable to the outcome of Experiment 8, the usual HVI would be maintained for the inverted scene, because the standards were on the same building wall.

\section{Method}

The observation was made binocularly. The subject wore goggles in which a right-angle prism was placed in front of each eye and was rotated within the frame of the goggles. The visual angle of the scene delimited by two prisms was $42.9^{\circ}$ vertically and $60.4^{\circ}$ horizontally. This implies that the visual field was greatly narrowed by the goggles, but its shape was still oval. The ratio of the vertical to horizontal extents of the visual field (.71) was approximately the same as that in the natural binocular viewing (.68).

In Experiments 6-7, the retinal image of the scene was tilted by $90^{\circ}$ by rotating the prisms. As a result, in Experiment 6 , in which the subjects were upright, the vertical standard along the building wall stimulated the horizontal retinal meridian and the horizontal standard stimulated the vertical retinal meridian. For half the subjects, the top of the scene was directed to the nasal side of the retina and for the remaining subjects, it was directed to the temporal side.

In Experiment 7, half the subjects lay on their right sides and the remaining subjects lay on their left sides. Nevertheless, by virtue of the goggles, by which the retinal image of the scene was rotated by $90^{\circ}$, the vertical standard stimulated the vertical meridian and the horizontal standard stimulated the horizontal meridian. That is, the top and base of the building stimulated the bottom and top of the retinal meridian, respectively. This retinal orientation was identical with that for the upright subjects under the natural binocular viewing.

In Experiment 8, in which the subject sat on a chair that was fixed steadily on a cart, the retinal image was rotated around the horizontal axis by tilting the prisms. Therefore, the scene was inverted as a whole (up-and-down inversion): The vertical and the horizontal standards stimulated the vertical and the horizontal meridians, respectively; but the top and base of the building stimulated the top and bottom of the retina, respectively.

\section{Results and Discussion}

Figure 4 shows the mean matched longitudinal distance against standard distance: The left panel represents the results for the upright subjects with the goggles, pro- 
ducing a $90^{\circ}$ tilt of the retinal image; the center panel represents the results for the lying-on-side subjects with the goggles producing the same tilt; and the right panel represents the results for the upright subjects with the goggles producing the up-and-down inversion. For each standard direction, a least-squares line passing through the origin was individually fitted to the matched distance as a function of standard distance.

For the upright subjects with the retinal image tilted, the mean slope for the vertical standards $(1.57, S D=$ .29) was significantly different $[t(13)=2.62, p<.05]$ from the mean slope for the horizontal standards (1.40, $S D=.23$ ). For the lying-on-side subjects with the retinal image tilted, the mean slope for the vertical standard (1.97, $S D=.22)$ was significantly different $[t(13)=2.22, p<$ $.05]$ from the mean slope for the horizontal standard $(1.81, S D=.23)$. For the upright subjects with the retinal image inverted, the mean slope for the vertical standard $(1.48, S D=.26)$ was not significantly different $[t(13)=2.10, .05<p<.10]$ from the mean slope for the horizontal standard $(1.40, S D=.27)$.

The results of Experiments 6-8 indicate that the HVI was greatly diminished by tilting the scene by $90^{\circ}$ or by inverting the scene. However, it seems difficult to find a suitable theory that accounts for this finding in the literature. It is not valid to assume that the reduction of the HVI effect in Experiments 6-8 was caused by the goggle frame restricting the visual field. We have already seen that narrowing the visual field by the cylinder did not have any effect on the HVI (Experiment 4).
One might assume that the prism goggles blurring the scene led to the reduction of the HVI. However, this assumption is not valid since the optical quality of scene was not greatly degraded by the prisms. Even if the prisms were slightly blurred, say, by moisture emitted from the face, no evidence demonstrates that two objects that differ only in clearness of detail appear to be at different distances (Rock, 1975). In addition, Coren and Girgus (1978) showed that the optical blur of the retinal image increased the amount of the visual illusions containing intersecting line elements, such as the MüllerLyer, but they did not show how the optical blur interacts with the HVI figure that contains fewer opportunities for contour interactions.

\section{COMPARISON WITH PREVIOUS THEORIES}

Tables 1-3 help us to compare the present results with the predictions from extant theories on the HVI. The first four rows of each table represent the predictions from the theories.

Table 1 shows the outcomes for the natural binocular viewing (Experiments 1-3). For the upright and the lyingon-belly subjects, any theory predicts the usual HVI. For the lying-on-side subjects, the physiology and frame theories predict the reversed HVI, and the depth and gravity theories predict the usual HVI. The obtained results did not agree with any theory.

Table 2 shows the outcomes for the cylindric viewing (Experiments 4-5). The depth and gravity theories pre-
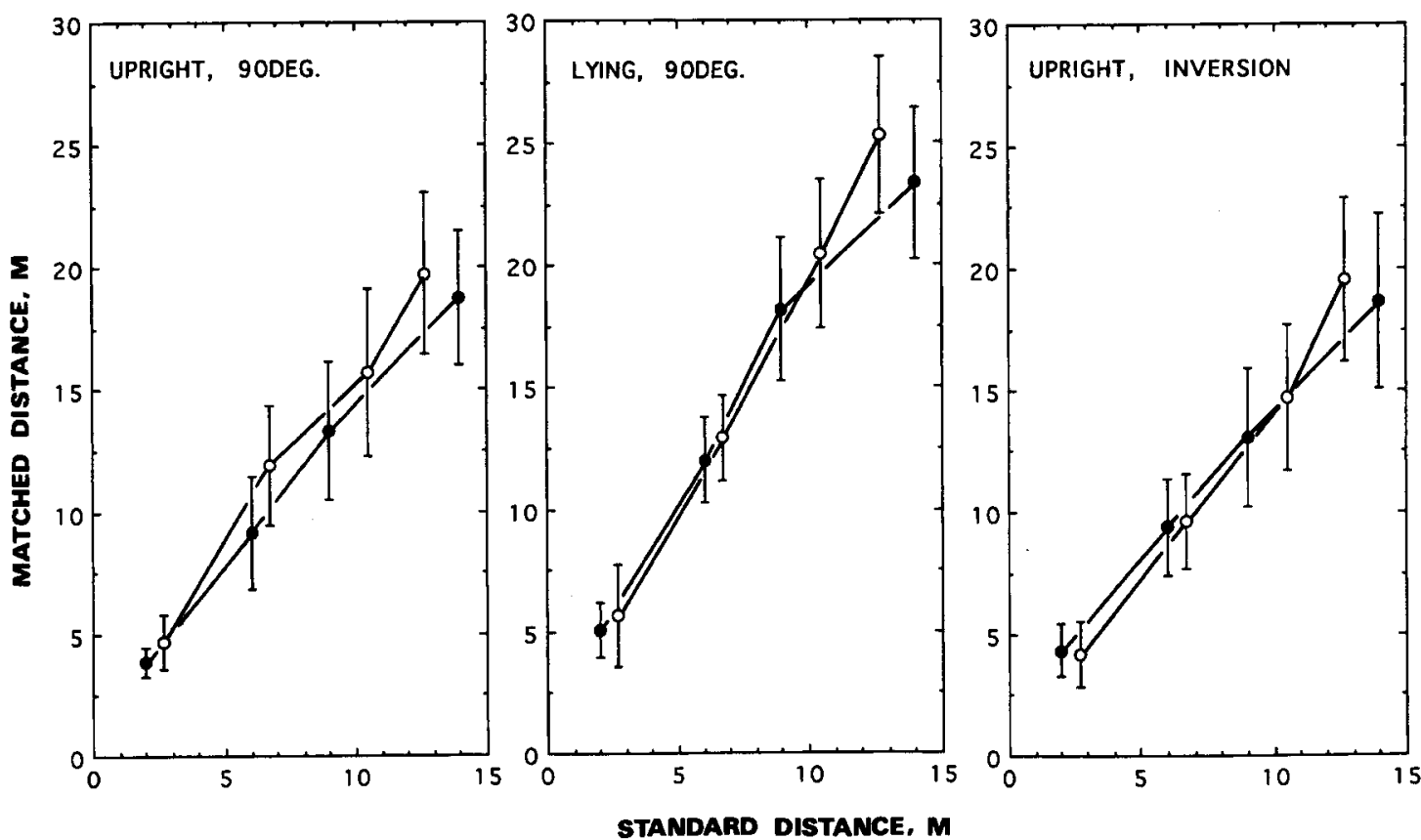

Figure 4. Mean longitudinal distance (in meters) as a function of standard distance under image-tilted conditions. The open circles represent the vertical standard and the filled circles represent the horizontal standard. The left panel is for the upright subjects seeing a $90^{\circ}$-tilted scene; the center panel for the lying-on-side subjects seeing a $90^{\circ}$-tilted scene; and the right panel for the upright subjects seeing an up-and-down-inverted scene. The bars passing through the data points represent the standard deviations. 
Table 1

Predictions From Four Theories and Amount of Illusion Obtained With Natural Binocular Viewings (Experiments 1-3)

\begin{tabular}{lccc}
\hline & \multicolumn{3}{c}{ Condition } \\
\cline { 2 - 4 } \multicolumn{1}{c}{ Model } & $\begin{array}{c}\text { Upright } \\
\text { (Experiment } 1)\end{array}$ & $\begin{array}{c}\text { Lying-on-Side } \\
\text { (Experiment 2) }\end{array}$ & $\begin{array}{c}\text { Lying-on-Belly } \\
\text { (Experiment 3) }\end{array}$ \\
Physiology & yes & reversed & yes \\
Frame & yes & reversed & yes \\
Depth & yes & yes & yes \\
Gravity & yes & yes & yes \\
Results & yes (1.38) & no (1.03) & yes (1.25) \\
\hline
\end{tabular}

Note-Yes indicates the usual HVI effect. No indicates that the vertical and horizontal standards are the same in adjustments of longitudinal distance. Reversed indicates the reversed HVI effect. The values in parentheses represent the amount of illusion (i.e., the ratio of the slope for the vertical to the slope for the horizontal standard).

dict the usual HVI for both the upright and the lying-onside subjects. On the contrary, the physiology theory predicts the usual HVI for the upright subjects and the reversed HVI for the lying-on-side subjects. The frame theory does not predict any illusion for either bodily orientation. The results did not agree with any theory.

Table 3 shows the outcomes for the image-tilted conditions (Experiments 6-8). For the upright subjects with the retinal image tilted, the depth theory predicts the usual HVI but other three theories predict the reversed HVI. For the lying-on-side subjects with the retinal image tilted, the gravity theory predicts the reversed HVI, but other theories predict the usual HVI. For the upright subjects with the retinal image inverted, all theories predict the usual HVI. Clearly, the results did not agree with these predictions.

Overall, no theory explains the results of Experiments 1-8 satisfactorily. One feature of the results is that the reversed HVI was not found at all, although some theories predict it in several conditions, as is indicated in Tables 1-3. Also, there appeared to be two classes with respect to the amount of illusion. In one class, as was obtained in Experiments 1, 3, and 4, the mean amount of illusion was 1.33 , which is three times or more that reported in most prior laboratory studies (the amount of illusion was $2 \%-10 \%$ according to Begelman \& Steinfeld, 1967, and Prinzmetal \& Gettleman, 1993). In another class, as was obtained in Experiments 6-7, the mean amount of illusion was 1.10 , which is greater than that typically obtained in laboratory settings.

\section{A MODEL}

Our model of distance perception consists of two main assumptions and several propositions. First, the HVI originates in a custom that has been formed in perceiving objects in the longitudinal plane. The retinal image that is projected by an object in the longitudinal plane differs from that by the same object in the frontal plane. That is, the retinal image for the longitudinal object is more shrunken along the vertical meridian relative to the horizontal meridian, but the retinal image for the frontal ob- ject is mostly faithful to the objective shape. For example, a square in the longitudinal plane yields a trapezoidal retinal image, whereas a square in the frontal plane produces a square retinal image. When a square in the longitudinal plane forms a trapezoid in the retina, we learn to realize that the retinal image represents, not a trapezoid, but a square, probably through touching the square directly (Berkeley, 1709/1910; Schwartz, 1994) or seeing it at the normal position in the frontal plane (James, 1890/1981). From birth onward, we frequently encounter such a relation between a retinal image and its representation, so perceiving the trapezoidal retinal image as a square is automatic. In other words, given a retinal image of a square, the dimension along the vertical meridian is perceived to be larger than that along the horizontal meridian. If this custom, which has been formed for the objects in the longitudinal plane, transfers to a square in the frontal plane, then the vertical dimension is perceived to be larger than the horizontal dimension of the same size.

Note that the compensatory custom for vertical distance in the frontal plane is formed while we hold our head upright. We sometimes look at the longitudinal and frontal planes with the heads tilted, but it is difficult to maintain such a tilted posture for a long time. Obviously, keeping the head upright is usually effortless. This does not mean

Table 2

Predictions From Four Theories and Amount of Illusion Obtained With Cylindric Viewings (Experiments 4-5)

\begin{tabular}{lcc}
\hline & \multicolumn{2}{c}{ Condition } \\
\cline { 2 - 3 } \multicolumn{1}{c}{ Model } & $\begin{array}{c}\text { Upright } \\
\text { (Experiment 4) }\end{array}$ & $\begin{array}{c}\text { Lying-on-Side } \\
\text { (Experiment 5) }\end{array}$ \\
\hline Physiology & yes & reversed \\
Frame & no & no \\
Depth & yes & yes \\
Gravity & yes & yes \\
Results & yes $(1.37)$ & no $(1.05)$ \\
\hline
\end{tabular}

Note - Yes indicates the usual HVI effect. No indicates that the vertical and horizontal standards are the same in adjustments of longitudinal distance. Reversed indicates the reversed HVI effect. The values in parentheses represent the amount of illusion (i.e., the ratio of the slope for the vertical to the slope for the horizontal standard).

Table 3

Predictions From Four Theories and Amount of Illusion Obtained With Image-Tilted Viewings (Experiments 6-8)

\begin{tabular}{|c|c|c|c|}
\hline \multirow[b]{2}{*}{ Model } & \multicolumn{3}{|c|}{ Condition } \\
\hline & $\begin{array}{c}\text { Upright } \\
90^{\circ} \text { Tilt } \\
\text { (Experiment 6) }\end{array}$ & $\begin{array}{c}\text { Lying-on-Side } \\
90^{\circ} \text { Tilt } \\
\text { (Experiment } 7 \text { ) }\end{array}$ & $\begin{array}{c}\text { Upright } \\
\text { Inversion } \\
\text { (Experiment } 8 \text { ) }\end{array}$ \\
\hline Physiology & reversed & yes & yes \\
\hline Frame & reversed & yes & yes \\
\hline Depth & yes & yes & yes \\
\hline Gravity & reversed & reversed & yes \\
\hline Results & yes (1.12) & yes $(1.09)$ & no $(1.05)$ \\
\hline
\end{tabular}

Note-Yes indicates the usual HVI effect. No indicates that the vertical and horizontal standards are the same in adjustments of longitudinal distance. Reversed indicates the reversed HVI effect. The values in parentheses represent the amount of illusion (i.e., the ratio of the slope for the vertical to the slope for the horizontal standard). 
that our heads are upright most of time. We constantly move our head; it is rare that the heads are upright even momentarily. However, the head position averaged over time is at least nearly upright and agrees with the gravitation. The HVI effect is assumed to be greatest possible in the usual way of viewing (Proposition 1). (The "usual way of viewing" refers to the situation in which the retinal orientation is the same as the visual and gravitational orientations.)

One might think that the compensatory custom could equally well take place for the horizontal direction, because we have seen the objects that slanted away from the frontal plane by rotating around the gravitational axis (e.g., the slanted wall of buildings). However, the compensatory custom seems stronger for the vertical direction than for the horizontal direction. Typically, we are on longitudinal planes such as the ground and the floor, and these longitudinal planes occupy a large portion of our visual field; in particular, the ground covers the lower half the visual field (Gibson, 1950). This implies that there are many instances for the compensatory process to work between two points in depth on the longitudinal plane. However, there is not a huge slanted vertical plane like the ground and there is no slanted vertical plane at sea or on the plains. This suggests that we have less chance of compensating for the shrinkage of horizontal distance. ${ }^{2}$

Second, the compensatory custom for vertical distance is assumed to be reduced or eliminated when there is a disagreement among the spatial orientations. Consequently, apparent horizontal and vertical distances are likely to be determined on the basis of the relation between the retinal image sizes, because the longitudinal distance up to the HVI figure is perceived to be constant. We assume that if the retinal orientation is discordant with both the visual and gravitational orientations, then the HVI disappears (Proposition 2). We also assume that if the retinal orientation agrees only with any other orientation, then the HVI effect may not disappear but is weakened (Proposition 3). Propositions 2 and 3 merely prescribe how the HVI effect in the frontal plane varies with the disagreement among the spatial orientations; they do not have implications for effectiveness of the linear perspective in the longitudinal plane, nor for the degree of size constancy.

It is useful to examine the results of Experiments 1-8 in conjunction with the predictions from the model. Proposition 1 predicts the greatest illusion effect for Experiments 1,3 , and 4 . Indeed, the obtained illusion was maximal in these experiments (1.33).

Proposition 2 predicts that the HVI would not occur in Experiments 2 and 5. Our results supported this prediction: The obtained illusion was very faint in these experiments (1.04).

Proposition 3 predicts a weak HVI effect for Experiments 6-7, because in Experiment 6, the retinal orientation was the same as the gravitational orientation and was orthogonal to the visual orientation; in Experiment 7, the retinal orientation was the same as the visual orientation and was orthogonal to the gravitational orientation. The obtained illusion was moderate in these experiments (1.11).

The prediction for Experiment 8 is not obtained directly from the model. However, since the usual way of viewing under which the perceptual custom has been formed was more greatly altered in Experiment 8 than in Experiment 7, the illusion effect might have been much more reduced in Experiment 8 . The illusion effect indeed disappeared in Experiment 8, but remained in Experiment 7 , though slight.

Our model does not predict the reversed HVI for any experiment of this study; it predicts three possible instances of strong, weak, and no HVI. This is in contrast with the other theories, assuming the reversed HVI in some specific conditions (in particular, in the situation in which the visual orientation was tilted $90^{\circ}$ under the usual retinal and gravitational orientations; Experiment 6 in Table 3).

One might also think that our model resembles the depth theory in that there is a perceptual coupling between the retinal image and its representation. However, in the depth theory, visual orientation is supposed to be a sole determinant of the HVI. Therefore, it is utterly impossible for the depth theory to predict how the visual orientation interacts with the retinal and gravitational orientations in the HVI effect.

In principle, our model is applicable not only to outdoor but to indoor situations, and it is capable of accounting for the small HVI effect that has been obtained for small objects at short viewing distances (e.g., Begelman \& Steinfeld, 1967; Prinzmetal \& Gettleman, 1993). In these situations, binocular convergence, retinal disparity, motion parallax, and other effects provide effective distance information up to the object. This distance information, together with the retinal size of the objects, is used to attain accurate size of the object (i.e., size constancy). This size-constancy process is supposed to overcome the compensatory process for vertical distance in the frontal plane.

The weak HVI obtained in the laboratory might also be related to the scantiness of stimuli suggesting the visual orientation. The illusion stimuli to be judged there were the meaningless geometric figures presented in otherwise darkness. These stimuli do not suggest strong visual orientation. In contrast, the outdoor situations contained ample stimuli suggesting the visual orientation (e.g., trees, buildings, and walking humans for the visual vertical, and the ground and floors for the visual horizontal). Since, in the absence of the visual orientation, the retinal orientation agrees only with the gravitational orientation, a weak HVI is predicted from Proposition 3.

\section{Mismatches of Longitudinal Distance}

Table 4 summarizes the mean slopes of linear functions obtained in this study and the mean visual angles of the standard distance subtended at the position where 
Table 4

Mean Slopes of Linear Functions Obtained in Experiments 1-8 and Mean Visual Angles (VA, in Degrees) of Standard Distance Subtended at Position Where Subjects Stopped

\begin{tabular}{cccccc}
\hline & \multicolumn{2}{c}{ Vertical } & & \multicolumn{2}{c}{ Horizontal } \\
\cline { 2 - 3 } \cline { 5 - 6 } Experiment & Slope & VA & & Slope & VA \\
\hline 1 & 1.57 & 32.5 & & 1.14 & 41.3 \\
2 & 1.35 & 36.5 & & 1.31 & 37.4 \\
3 & 1.30 & 30.3 & 1.04 & 38.7 \\
4 & 1.71 & 30.3 & 1.25 & 38.7 \\
5 & 1.94 & 27.3 & 1.84 & 28.5 \\
6 & 1.57 & 32.5 & 1.40 & 35.5 \\
7 & 1.97 & 26.9 & 1.81 & 28.9 \\
8 & 1.48 & 32.4 & 1.40 & 35.5 \\
\hline
\end{tabular}

the subjects stopped (i.e., a cotangent of slope). If the visual system exerted like a survey instrument, the slope would be unity and the visual angle would be $45^{\circ}$. The obtained slopes were consistently larger than unity and the visual angles were therefore smaller than $45^{\circ}$.

Since the ratio of the apparent frontal to apparent longitudinal distances was kept equal to unity in this study, the results may be formulated as

$$
\frac{S^{\prime}}{D^{\prime}}>\tan \theta
$$

where $S^{\prime}$ is the apparent frontal distance, $D^{\prime}$ is the apparent longitudinal distannce, and $\theta$ is the visual angle. This result parallels that in the Higashiyama and Shimono (1994) study, in which $S^{\prime} / D^{\prime}$ was found to be a power function of apparent visual angle, larger than its actual visual angle.

The tendency to adjust longitudinal distance mistakenly was remarkable for the vertical standard. In particular, the slope obtained for the upright subjects was 1.57 for the natural binocular condition and 1.71 for the cylindric condition. These slopes compared with the value of 1.60 that was obtained for different subjects in different situations (Higashiyama \& Ueyama, 1988).

The mismatches of longitudinal distance were also obtained for the horizontal standards. Table 4 indicates that the matched longitudinal distances were shown to be 1.14-1.84 times larger than the horizontal standards. Foley (1972) first reported that longitudinal distance matches exceeded horizontal distances in a situation in which only binocular convergence and disparity were available. In a natural full-cue situation as well, depth that was matched so as to appear equal to the horizontal standard was larger than the standard (Loomis, Da Silva, Fujita, \& Fukushima, 1992). The matched depth was shown to be 1.2-2.0 times larger than the standard. Using magnitude estimation, category estimation, and mapping, Wagner (1985) showed that estimates of spatial extent were described by a power function with an exponent of about 1.0, but the scale factor for extent in depth was about half as large as that for extent in the frontal plane. These studies imply that extent seen in depth tends to be perceptually contracted relative to that in the frontal plane.
Why did the longitudinal distance matches exceed the frontal distances in this study? One factor is the reduction of cues to distances. It is well documented that as cues to distance are reduced, the relation between apparent extents is likely to be determined by the relation between the retinal image sizes. In the situation in which a frontal distance is objectively equal to a longitudinal distance, the frontal distance subtends a larger visual angle than does the longitudinal distance. Despite the difference of visual angles, the two distances are perceived as being equal to each other in the usual way of viewing with full cues to distance. However, when cues to distance are reduced, because we are apt to respond to the relation between retinal image sizes, the longitudinal distance is perceived to be smaller than the frontal distance. Thus, the longitudinal distance has to be enlarged to appear equal to the frontal distance.

In this study, reducing cues to distance may be attained by lowering the point of view (Higashiyama \& Ueyama, 1988 ) and by truncating the visual field with the cylinder or goggles (Hagen et al., 1978; Holway \& Boring, 1941). Table 5 shows how each experiment contained the factors reducing cues to distance. The filled circles in Figure 5 show a relation between the slope for the horizontal standard (Table 4) and the degree of cue reduction (Table 5).

Another factor affecting the mismatches of longitudinal distance is the HVI effect. Because the HVI is as-

Table 5

Factors Affecting Mismatch of Longitudinal Distance for Horizontal Standard

\begin{tabular}{cccc}
\hline Experiment & $\begin{array}{c}\text { Low Point of } \\
\text { View }\end{array}$ & $\begin{array}{c}\text { Truncated } \\
\text { View }\end{array}$ & Total \\
\hline 1 & & & 0 \\
2 & + & & 1 \\
3 & + & + & 1 \\
4 & & + & 1 \\
5 & + & + & 2 \\
6 & + & + & 1 \\
7 & + & + & 1 \\
8 & & + & 1 \\
\hline
\end{tabular}

Note-Plus signs indicate factor reducing cues to distance present. For the sake of simplicity, the degree of cue reduction in each experiment is represented by the number of pluses.

Table 6

Factors Affecting Mismatch of Longitudinal Distance for Vertical Standard

\begin{tabular}{ccccc}
\hline Experiment & $\begin{array}{c}\text { Low Point of } \\
\text { View }\end{array}$ & $\begin{array}{c}\text { Truncated } \\
\text { View }\end{array}$ & $\begin{array}{c}\text { HVI } \\
\text { Effect }\end{array}$ & Total \\
\hline 1 & & & + & 1 \\
2 & + & & + & 1 \\
3 & + & + & + & 2 \\
4 & + & + & & 2 \\
5 & + & + & + & 2 \\
6 & + & + & + & 3 \\
7 & & + & & 1 \\
8 & & & & \\
\hline
\end{tabular}

Note - For the sake of simplicity, the combinational effect of the three factors is represented by the total number of pluses. 


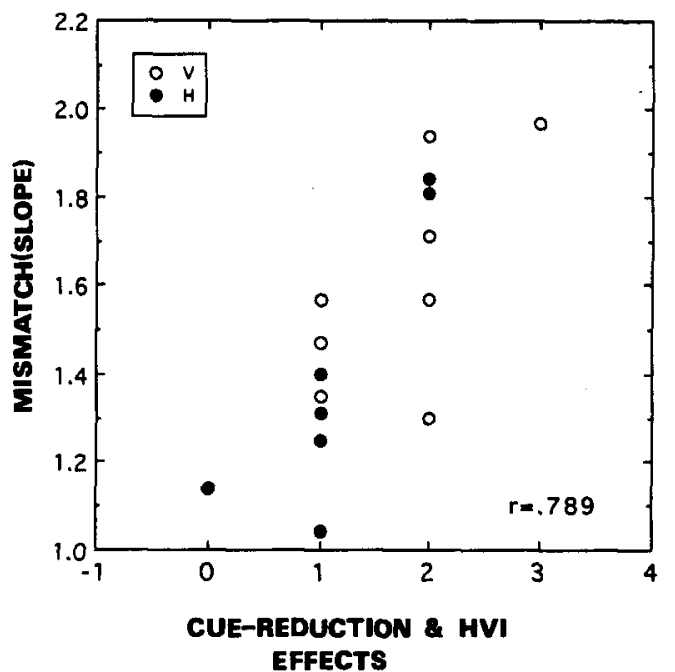

Figure 5. The mismatch of longitudinal distance as a function of combination of the cue-reduction effect and the HVI effect. The open circles represent the vertical standard and the filled circles represent the horizontal standard. The ordinate 1.0 represents the precise matches of longitudinal distance.

sumed to result from compensating for the shrinkage of the vertical dimension, it is supposed that the longitudinal distance matches exceed the frontal distances much more for the vertical standard than for the horizontal standard.

The combination of the cue-reduction and HVI effects may be correlated with the mismatch of longitudinal distance for the vertical standard. Table 6 shows how each experiment contained the HVI factor as well as the cuereduction factors. A relation between the mismatch for the vertical standard and the combinational effect is shown by open circles in Figure 5. The correlation for all points in Figure 5 was 0.789 , significantly different from zero $(n=16, p<.05)$. Although the obtained correlation may be far from perfect, the three variables clearly contributed to the use of greater longitudinal distances to match frontal distances.

\section{REFERENCES}

AVERY, G. C., \& DAY, R. H. (1969). Basis of the horizontal-vertical illusion. Journal of Experimental Psychology, 81, 376-380.

Begelman, D. A., \& Steinfeld, G. (1967). An investigation of several parameters of the horizontal-vertical illusion. Perception \& Psychophysics, 2, 539-543.

BERKELEY, G. (1910). An essay towards a new theory of vision. New York: E. P. Dutton. (Original work published 1709)

BORING, E. G. (1942). Sensation and perception in the history of experimental psychology. New York: Appleton-Century-Crofts.

Bower, T. G. R. (1966, December). The visual world of infants. Scientific American, 215, 80-92.

Brislin, R. W., \& Leibowitz, H. W. (1970). The effect of separation between test and comparison objects on size constancy at various age-levels. American Journal of Psychology, 83, 372-376.

CAELLI, T. (1977). Is perceived length affected by interactions between orientation detectors? Vision Research, 17, 837-841.

Chapanis, A., \& Mankin, D. A. (1967). The vertical-horizontal illu- sion in a visually-rich environment. Perception \& Psychophysics, 2, 249-255.

COREN, S., \& GiRgus, J. S. (1977). Illusions and constancies. In W. Epstein (Ed.), Stability and constancy in visual perception: Mechanisms and processes (pp. 255-283). New York: Wiley.

Coren, S., \& Girgus, J. S. (1978). Seeing is deceiving: The psychology of visual illusion. Hillsdale, NJ: Erlbaum.

Cormack, E. O., \& Cormack, R. H. (1974). Stimulus configuration and line orientation in the horizontal-vertical illusion. Perception \& Psychophysics, 16, 208-212.

DA Silva, J. A. (1985). Scales for perceived egocentric distance in a large open field: Comparison of three psychophysical methods. American Journal of Psychology, 98, 119-144.

FOLEY, J. M. (1972). The size-distance relation and intrinsic geometry of visual space: Implications for processing. Vision Research, 12, 323-332.

GiBson, J. J. (1950). The perception of the visual world. Boston: Houghton Mifflin.

Girgus, J. S., \& COREN, S. (1975). Depth cues and constancy scaling on the horizontal-vertical illusion: The bisection error. Canadian Journal of Psychology, 29, 59-65.

GREGORY, R. L. (1963). Distortion of visual space as inappropriate constancy scaling. Nature, 199, 678-780.

Gregory, R. L. (1972). Eye and brain (2nd ed.). New York: McGrawHill.

HAGEN, M. A., JoNES, R. K., \& REed, E. S. (1978). On a neglected variable in theories of pictorial perception: Truncation of the visual field. Perception \& Psychophysics, 23, 326-330.

Henson, D. V. (1993). Visual fields. Oxford: Oxford University Press. HigashiYama, A. (1992). Anisotropic perception of visual angle: Implications for the horizontal-vertical illusion, overconstancy of size, and the moon illusion. Perception \& Psychophysics, 51, 218-230.

Higashiyama, A., Ishikawa, T., \& TanaKa, K. (1990). Visual alleys as a function of instructions under informative and reduced conditions of viewing. Perception \& Psychophysics, 47, 468-476.

Higashiyama, A., \& Shimono, K. (1994). How accurate is size and distance perception for very far terrestrial objects? Function and causality. Perception \& Psychophysics, 55, 429-442.

Higashiyama, A., \& Ueyama, E. (1988). The perception of vertical and horizontal distances in outdoor settings. Perception \& Psychophysics, 44, 151-156.

HOLWAY, A. H., \& BorING, E. G. (1941). Determinants of apparent visual size with distance variant. American Journal of Psychology, 54, 21-37.

Houck, R. L., Mefferd, R. B., \& Greenstein, G. J. (1972). Influence of a visual frame and vertical-horizontal illusion on shape and size perception. Journal of Experimental Psychology, 96, 273-279.

Howard, I. P. (1982). Human visual orientation. New York: Wiley.

HoWARD, I. P., \& TEMPLETON, W. B. (1966). Human spatial orientation. New York: Wiley.

JAMES, W. (1981). Principles of psychology. Cambridge, MA: Harvard University Press. (Original work published 1890)

KÖHLER, W. (1940). Dynamics in psychology. New York: Liveright.

KüNNAPAS, T. M. (1955). Influence of frame size on apparent length of a line. Journal of Experimental Psychology, 50, 168-170.

KÜNNAPAS, T. M. (1957a). Interocular differences in the verticalhorizontal illusion. Acta Psychologica, 13, 253-259.

KüNNAPAS, T. M. (1957b). Vertical-horizontal illusion and the surrounding field. Acta Psychologica, 13, 35-42.

KüNNAPAS, T. M. (1957c). The vertical-horizontal illusion and the visual field. Journal of Experimental Psychology, 53, 405-407.

KÜNNAPAS, T. M. (1958). Influence of head inclination on the verticalhorizontal illusion. Journal of Psychology, 46, 179-185.

KüNNAPAS, T. M. (1959). The vertical-horizontal illusion in artificial visual fields. Journal of Psychology, 47, 41-48.

Leibowitz, H. W., Pollard, S. W., \& Dickson, D. (1967). Monocular and binocular size matching as a function of distance at various age levels. American Journal of Psychology, 80, 263-268.

Loomis, J. M., Da Silva, J. A., Fuitta, N., \& Fukushima, S. S. (1992). Visual space perception and visually directed action. Journal of Experimental Psychology: Human Perception \& Performance, 18, 906 921. 
LUCKIESH, M. (1965). Visual illusion. New York: Dover.

Miyagawa, T. (1943). Toritsu-shi ni kansuru jikkenteki kenkyu: Iwayuru mata nozoki no mondai [An experimental study of scenes seen between the legs with the head upside down]. Japanese Journal of Psychology, 18, 289-309.

PearCe, D., \& Matin, L. (1969). Variation of the magnitude of the horizontal-vertical illusion with retinal eccentricity. Perception \& Psychophysics, 6, 241-243.

Prinzmetal, W., \& Gettleman, L. (1993). Vertical-horizontal illusion: One eye is better than two. Perception \& Psychophysics, 53, 81-88.

RestLe, F. (1970, February 20). Moon illusion explained on the basis of relative size. Science, 167, 1092-1096.

RESTLE, F., \& MERrYMan, C. (1969). Distance and an illusion of length of line. Journal of Experimental Psychology, 81, 297-302.

RitTer, S. M. (1917). The vertical-horizontal illusion. Psychological Monographs (Whole No. 101).

Rock, I. (1973). Orientation and form. New York: Academic Press. Rock, I. (1975). An introduction to perception. New York: Macmillan. Rock, I., \& Kaufman, L. (1962, June 22). The moon illusion, II. Science, 136, 1023-1031.

Schiffman, H. R., \& Thompson, J. G. (1975). The role of figure orientation and apparent depth in the perception of the horizontalvertical illusion. Perception, 4, 79-83.

SCHWARTZ, R. (1994). Vision: Variations on some Berkeleian themes. Cambridge: Blackwell.

Thompson, J. G., \& SchiffMAn, H. R. (1974a). The effect on the magnitude of the horizontal--vertical illusion of horizontal retinal eccentricity. Vision Research, 14, 1463-1465.

Thompson, J. G., \& Schiffman, H. R. (1974b). The influence of figure size and orientation on the magnitude of the horizontal-vertical illusion. Acta Psychologica, 38, 413-420.

VALENTINE, C. W. (1912a). The effect of astigmatism on the horizontal- vertical illusion, and a suggested theory of the illusion. British Journal of Psychology, 5, 308-330.

VAlentine, C. W. (1912b). Psychological theories of the horizontalvertical illusion. British Journal of Psychology, 5, 8-35.

VERRILLo, R. T., \& IRVIN, G. (1979). Absolute estimation of line length as a function of orientation and contrast polarity. Sensory Processes, 3, 261-274.

von Collani, G. (1985). The horizontal-vertical illusion in photographs of concrete scenes with and without depth information. Perceptual \& Motor Skills, 61, 523-531.

Wagner, M. (1985). The metric of visual space. Perception \& Psychophysics, 38, 483-495.

\section{NOTES}

1. The outcomes of Experiments 6-8 are clear. Nevertheless, inspection of Figure 4 might lead one to doubt the significant differences among the results of the experiments. We should not forget, however, that statistical decisions have been made to avoid the kind of confusion that might arise on the basis of simple visual inspection.

2. It is possible that the tendency to compensate for shrinkage of vertical distance is innate. Even in this case, this tendency is probably reinforced through visual and haptic contacts with objects and formed as a powerful perceptual skill. The studies of size constancy, for example, clearly demonstrate a general relation between innate perceptual ability and acquired perceptual skill. A 6- to 8-week-old infant is likely to perceive the size of an object to be constant independent of viewing distance (e.g., Bower, 1966). Yet the story of size constancy does not stop in infancy; it is clear that size constancy develops over a long period (e.g., Brislin \& Leibowitz, 1970; Leibowitz, Pollard, \& Dickson, 1967).

(Manuscript received February 27, 1995; revision accepted for publication July 31,1995 .) 